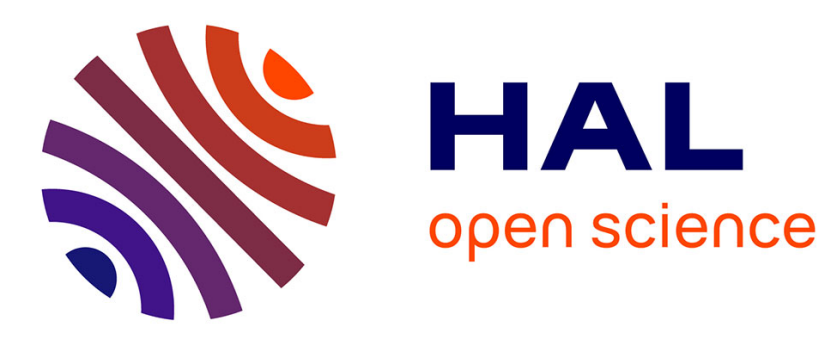

\title{
Material decomposition mechanisms in femtosecond laser interactions with metals
}

Mikhail E. Povarnitsyn, Tatiana Itina, Marc Sentis, Konstantine Khishchenko, Pavel Levashov

\section{- To cite this version:}

Mikhail E. Povarnitsyn, Tatiana Itina, Marc Sentis, Konstantine Khishchenko, Pavel Levashov. Material decomposition mechanisms in femtosecond laser interactions with metals. Physical Review B: Condensed Matter and Materials Physics (1998-2015), 2007, 75 (23), pp.235414. 10.1103/PhysRevB.75.235414 . hal-00194823

\section{HAL Id: hal-00194823 \\ https://hal.science/hal-00194823}

Submitted on 22 Sep 2021

HAL is a multi-disciplinary open access archive for the deposit and dissemination of scientific research documents, whether they are published or not. The documents may come from teaching and research institutions in France or abroad, or from public or private research centers.
L'archive ouverte pluridisciplinaire HAL, est destinée au dépôt et à la diffusion de documents scientifiques de niveau recherche, publiés ou non, émanant des établissements d'enseignement et de recherche français ou étrangers, des laboratoires publics ou privés. 


\title{
Material decomposition mechanisms in femtosecond laser interactions with metals
}

\author{
M. E. Povarnitsyn, ${ }^{1,2}$ T. E. Itina, ${ }^{1}$ M. Sentis, ${ }^{1}$ K. V. Khishchenko, ${ }^{2}$ and P. R. Levashov ${ }^{2}$ \\ ${ }^{1}$ Laboratory of Lasers, Plasmas and Photonic Processing (LP3), UMR 6182 CNRS, 163 avenue de Luminy, Case 917, \\ 13288 Marseille, France \\ ${ }^{2}$ Institute for High Energy Densities, RAS, Izhorskaya 13/19, Moscow 125412, Russia
}

(Received 14 February 2007; published 12 June 2007)

\begin{abstract}
A numerical hydrodynamic study of femtosecond laser ablation is presented. A detailed analysis of material decomposition is performed using a thermodynamically complete equation of state with separate stable and metastable phase states and phase boundaries. The lifetime of the metastable liquid state is estimated based on the classical theory of homogeneous nucleation. In addition, mechanical fragmentation of the target material is controlled based on available criteria. As a result, several ablation mechanisms are observed. A major fraction of the ablated material, however, is found to originate from the metastable liquid region, which is decomposed either thermally in the vicinity of the critical point into a liquid-gas-mixture or mechanically at high strain rate and negative pressure into liquid droplets and chunks. The calculation results explain available experimental findings.
\end{abstract}

DOI: 10.1103/PhysRevB.75.235414 PACS number(s): 61.80.Az, 64.70.Dv, 64.70.Fx, 79.20.Ds

\section{INTRODUCTION}

Femtosecond laser interactions have attracted a particular interest of researchers during the last decade. It is not surprising since these new ultrashort laser pulses provide a unique opportunity for the fast development of numerous very promising and exciting applications, such as laser machining, micro- and nanostructuring, laser-induced plasma spectroscopy, nanoparticle synthesis in vacuum, in gas, or in a liquid solution, medical imaging, laser surgery, etc. ${ }^{1-3}$ Many experimental studies were performed, demonstrating the unique possibilities of femtosecond laser systems. ${ }^{4-6} \mathrm{At}$ the same time, several theoretical investigations were focused on the better understanding of physical processes involved in ultrashort laser interactions. ${ }^{7-21}$ The classical approach typically used for these interactions is based on a two-temperature model. ${ }^{13}$ In addition, several hydrodynamic simulations were carried out to describe the target material motion. ${ }^{10,11,14-16}$ The formation of a thermal wave, pressure gain, and shock wave propagation have been observed in these calculations. Molecular dynamics (MD) simulations were furthermore performed that provided insights into the mechanisms, such as phase explosion, fragmentation, evaporation, and mechanical spallation. ${ }^{17-21}$

In spite of a significant effort aimed at the understanding of the ablation mechanisms, several issues stay unclear. For example, theoretically predicted ablation depth was frequently underestimated with respect to the typical experimental values. This result is an indication that not all the ablation mechanisms were taken into account. In addition, numerous terms used to describe these mechanisms are confusing or misused. For instance, when an explosive boiling term is used, which reflects the overheated (metastable) liquid decomposition into a liquid-gas mixture, it is often associated with material heating. The metastable liquid state, however, can also be achieved during fast material expansion and cooling. The proper term for this process is unclear. In this case, critical-point phase separation was proposed in Ref. 15 but only to describe material decomposition in the vicinity of the critical point. In addition, up to now, it was difficult to handle this process correctly in the framework of hydrodynamic models because most of the available equations of state (EOSs) did not describe the metastable regions. Moreover, no kinetics of gas bubble formation or/and condensation was previously introduced to determine the lifetime of these states. Besides, there is still a lack of information about the photomechanical failure of the material at extremely high strain rates (up to $10^{9} \mathrm{~s}^{-1}$ ), which can be achieved. ${ }^{7}$ Nevertheless, even these high strain rates do not always satisfy the fragmentation criteria ${ }^{22,23}$ because of their short duration (tens of picoseconds). All these issues need more analysis.

\section{MODEL}

To bring more light on the mechanisms of material decomposition in femtosecond laser ablation of metals, we performed a detailed hydrodynamic modeling. Note that compared to atomistic techniques, hydrodynamic models allow calculations for much larger systems and take much shorter computer time. Our numerical model is based on the solution of a system of Eulerian hydrodynamic equations by a highorder multimaterial Godunov's method. ${ }^{24}$ The equations were extended to the case of one-fluid two-temperature hydrodynamics with laser energy absorption source, electron heat conductivity, and energy exchange between electrons and heavy particles (atoms, ions, nuclei). ${ }^{25}$ A Gaussian temporal profile is used to simulate the laser energy deposition. The heat conductivity of electrons is calculated according to the model. ${ }^{26}$ Energy exchange between electrons and heavy particles, the reflectivity coefficient $R$, and the optical penetration depth $\lambda_{\text {opt }}$ are derived by means of the wide-range frequency of electron-phonon and/or electron-ion collisions. ${ }^{14}$

For completeness of our model, we use a semiempirical multiphase EOS for aluminum with separate description of subsystems of heavy particles and electrons. The specific Helmholtz free energy has a form $\mathcal{F}\left(\rho, T_{i}, T_{e}\right)=\mathcal{F}_{i}\left(\rho, T_{i}\right)$ 
$+\mathcal{F}_{e}\left(\rho, T_{e}\right)$, composed of two parts which describe the contributions of heavy particles and electrons, respectively. Here, $\rho$ is the material density and $T_{i}$ and $T_{e}$ are temperatures of heavy particles and electrons. The first item $\mathcal{F}_{i}\left(\rho, T_{i}\right)$ $=\mathcal{F}_{c}(\rho)+\mathcal{F}_{a}\left(\rho, T_{i}\right)$, in turn, consists of the electron-ion interaction term $\mathcal{F}_{c}$ (calculated at $T_{i}=T_{e}=0 \mathrm{~K}$ ) and the contribution of thermal motion of heavy particles $\mathcal{F}_{a}$. Analytical form of $\mathcal{F}_{i}$ has different expressions for the solid $\mathcal{F}_{i}^{(s)}$, as well as for both the liquid and gas phases $\mathcal{F}_{i}^{(l)} .{ }^{27}$ Using these thermodynamic functions, the solid, liquid, and gas phase equilibrium boundaries are determined from the equality conditions for the temperature $T_{i}$, pressure $P_{i}=\rho^{2}\left(\partial \mathcal{F}_{i} / \partial \rho\right)_{T_{i}}$, and Gibbs potential $G_{i}=\mathcal{F}_{i}+P_{i} / \rho$ of each phase pair. ${ }^{28}$ The tables of thermodynamic parameters are calculated taking into consideration the information about phase transitions and metastable regions..$^{29,30}$ The free energy of electrons in metal $\mathcal{F}_{e}$ has a finite-temperature ideal Fermi-gas form. ${ }^{28}$

The model of our EOS contains about 40 adjustable constants so that the EOS meets the following requirements: (i) to describe experimental results on compression and expansion for a wide range of densities and temperatures including data on critical and triple points; (ii) to contain separate information about electron and ion (or lattice) subsystems; and (iii) to represent changes of thermodynamic parameters during phase transitions. To account for kinetic processes, an estimation of the realistic lifetime of metastable liquid state is introduced. Using this lifetime-based information, we switch between two different modifications of the EOS: (i) with metastable states and (ii) without metastable states.

In our model, when the liquid branch of the binodal curve was crossed and the matter turned into metastable state, we include a particular treatment for each of the following two competitive effects: (i) for the thermal decomposition, a criterion of the metastable liquid lifetime is used based on the theory of homogeneous nucleation; ${ }^{31-33}$ (ii) for the mechanical fragmentation, a failure criterion of Grady is applied. ${ }^{22}$

In the first case, we estimate the metastable liquid lifetime as $\tau_{\text {nucl }}=(C n V)^{-1} \exp \left(W / k_{B} T_{i}\right)$, where $C=10^{10} \mathrm{~s}^{-1}$ is the kinetic coefficient, ${ }^{33} n$ is the concentration, $V$ is the volume under consideration (the volume of numerical cell in our case), $W=16 \pi \sigma^{3} / 3 \Delta P^{2}$ is the work required on a gas bubble formation in a liquid phase, $k_{B}$ is the Boltzmann constant, and $\Delta P$ is the difference between the saturated vapor pressure (known from EOS) and the pressure of substance. The temperature dependence of the surface tension $\sigma$ is described in the form $\sigma=\sigma_{0}\left(1-T_{i} / T_{c}\right)^{1.25}$, (see Refs. 33 and 34). Here, $T_{c}=6595 \mathrm{~K}$ is the temperature in the critical point $(\mathrm{CP})$ for $\mathrm{Al}$ (Ref. 27) and $\sigma_{0}=860 \mathrm{~g} / \mathrm{s}^{2}$ is the surface tension of $\mathrm{Al}$ at melting temperature. ${ }^{34}$ In this case, as soon as the lifetime $\tau_{\text {nucl }}$ in the volume $V$ is expired, the metastable one-phase state separates into two-phase stable liquid-gas mixture. This phase separation process is accompanied by abrupt changes of thermodynamic parameters such as pressure, temperature, sound speed, compressibility, heat capacity, etc. The EOS with metastable phase states is therefore no more relevant in this volume, so that we continue to calculate the thermodynamic properties by using the EOS without metastable states.

To account for the second effect, a fragmentation criterion $^{22}$ is used for the liquid phase with the spall strength

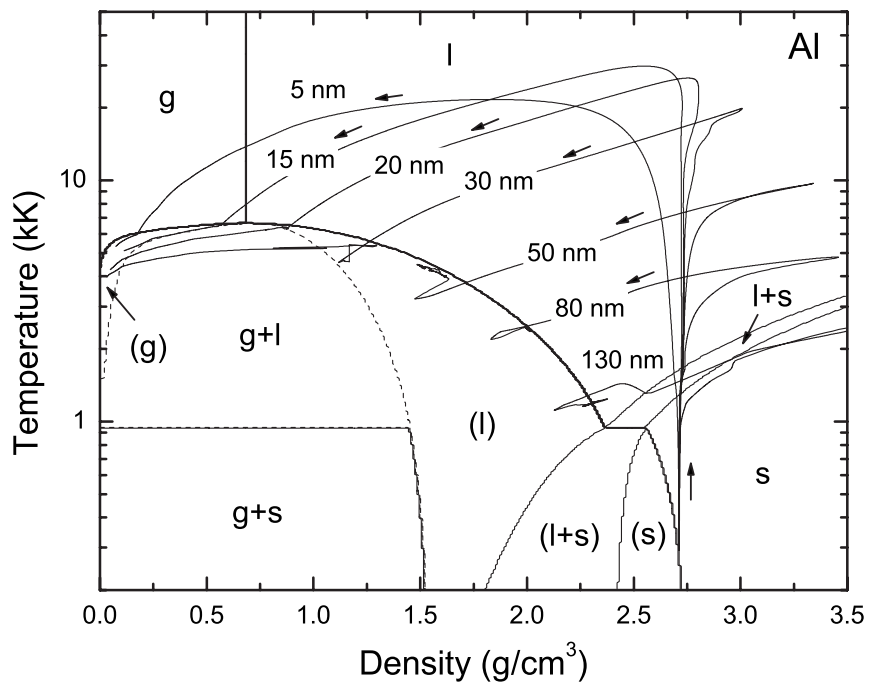

FIG. 1. Phase diagram of aluminum and time evolution of density and temperature of heavy particles for different layers of the target. Dashed curve, the spinodal; g, stable gas; 1, stable liquid; s, stable solid; $1+\mathrm{s}$, stable melting; $\mathrm{g}+\mathrm{l}$, liquid-gas mixture; $\mathrm{g}+\mathrm{s}$ : solid-gas mixture; (g), metastable gas; (1), metastable liquid; $(1+\mathrm{s})$, metastable melting; (s), metastable solid. The phase trajectories correspond to depths of $5,15,20,30,50,80$, and $130 \mathrm{~nm}$ from the initial target surface. Arrows along the trajectories show the flow of time. Here, laser pulse parameters are $\tau_{L}=100 \mathrm{fs}, \lambda_{L}=800 \mathrm{~nm}$, and $F=5 \mathrm{~J} / \mathrm{cm}^{2}$.

$P_{\text {spal }}=\left(6 \rho^{2} c^{3} \sigma \dot{\varepsilon}\right)^{1 / 3}$ and the time to fracture $\tau_{\text {spal }}$ $=\left(6 \sigma / \rho \dot{\varepsilon}^{2} c^{3}\right)^{1 / 3}$, where $\dot{\varepsilon}$ is the strain rate and $c$ is the sound speed. When the pressure of matter drops below the negative value $-P_{\text {spal }}$ and the duration of this event is longer than the time $\tau_{\text {spal }}$, a criterion of fragmentation is satisfied and voids and new free surfaces can appear in the substance. To describe this phenomenon numerically we let a "broken" substance shrink back until the pressure comes to zero value. We compensate the difference between the old and new volumes by introducing void fraction into a numerical cell. Both thermal and mechanical criteria described above are used simultaneously and each of them can prevail in a given computational cell depending on the substance location on the phase diagram.

\section{RESULTS}

For the analysis of the target material evolution after the laser irradiation, it is convenient to use a phase diagram in the temperature-density plane $(T, \rho)$ given by the EOS (Fig. $1)$. The binodal curve corresponds to the liquid-vapor coexistence line (saturated vapor curve). The spinodal curve (dashed) gives a limit of thermodynamic stability of the liquid and gas phases. The regions between the binodal and the spinodal curves show the metastable states of matter: the superheated liquid (1) and the supercooled vapor (g) states.

Initially, the aluminum target is in a solid state with $\rho$ $=2.71 \mathrm{~g} / \mathrm{cm}^{3}$ and $T_{i}=T_{e}=300 \mathrm{~K}$, and is subjected to a laser pulse with fluence $F=5 \mathrm{~J} / \mathrm{cm}^{2}$, pulse width $\tau_{L}=100 \mathrm{fs}$, and wavelength $\lambda_{L}=800 \mathrm{~nm}$. The reflectivity coefficient and the 
optical penetration depth are calculated ${ }^{14}$ and their values are $R=0.92$ and $\lambda_{o p t}=13 \mathrm{~nm}$, respectively. The conduction-band electrons absorb laser energy in a skin layer (a very thin region of the order of the optical penetration depth $\lambda_{\text {opt }}$ ). Then, these hot electrons transmit absorbed energy into the bulk owing to heat conduction and warm up the lattice due to electron-phonon collisions. The time of electron-lattice relaxation (equilibration of temperatures of electron and heavy particle subsystems) is of the order of several picoseconds for aluminum and thus the lattice pressure has time to grow up before the heat energy of electrons dissipates into the bulk of the target. Formation of a narrow zone with a sharp pressure profile results in generation of intensive shock wave, which moves into the bulk, and rarefaction wave that causes a spread of the matter out of the target. The process of the shock wave propagation is clearly visible as the consecutive right-hand deviations of the $30,50,80$, and $130 \mathrm{~nm}$ trajectories in Fig. 1. The process of compression of different target layers changes into an expansion process (decreasing of the density along trajectories, Fig. 1).

The maximal temperature of heavy particles in the vicinity of the target surface (depth $\leqslant 15 \mathrm{~nm}$ ) is high enough (up to $T_{i} \sim 30 \mathrm{kK}$ ) so that the phase trajectories from this layer go above the $\mathrm{CP}$ and the target material is directly transformed into the gas phase (atomization, 5 and $15 \mathrm{~nm}$ trajectories in Fig. 1). Then, these trajectories cross the gas branch of the binodal and penetrate into the supercooled vapor region, where condensation starts and liquid-gas mixture forms. Note, however, that this layer represents a very small fraction of the ablated material so that the condensation degree is insignificant.

The next layer in the target (depth from 20 to $30 \mathrm{~nm}$ ) is first transformed into a metastable liquid state. This layer is heated to high temperatures (up to $T_{i} \sim 25 \mathrm{kK}$ ) so that the corresponding phase trajectories cross the liquid branch of binodal in the vicinity of the $\mathrm{CP}$ and enter the metastable liquid region. Under these conditions, the target material is thermodynamically unstable. The lifetime of this state is estimated as described above. Thermodynamic instabilities are known to occur near the $\mathrm{CP}$ (particularly, at $0.9 T_{c}<T_{i}<T_{c}$ ), leading to a rapid decomposition (several picoseconds) of the matter into a liquid-gas mixture. This process is similar to the phase explosion, ${ }^{17,18}$ though it occurs during simultaneous material expansion and cooling. The other term used for this mechanism is the critical-point phase separation proposed in Ref. 15.

This thermal mechanism, however, concerns only quite a thin slice ( $\sim 10 \mathrm{~nm}$ thickness), which trajectories enter the metastable liquid region near the critical point. In fact, the melted layer is much thicker $\left(\sim 200 \mathrm{~nm}\right.$ at $\left.F=5 \mathrm{~J} / \mathrm{cm}^{2}\right)$. The rest of the trajectories (from 50 to $130 \mathrm{~nm}$ in Fig. 1), which originate from the deeper lying melted layers of the target, also enter the metastable liquid region, but at temperatures much smaller than the critical temperature $T_{c}$. In this case, the matter can stay in the metastable state much longer. As a result, mechanical effects start to dominate over the thermal ones and material fragmentation (cavitation in liquid) occurs under the action of a tensile pressure wave. In other words, the time to mechanical fracture is shorter than the nucleation time $\left(\tau_{\text {nucl }}<\tau_{\text {spal }}\right)$. As soon as the time to fracture is ex-
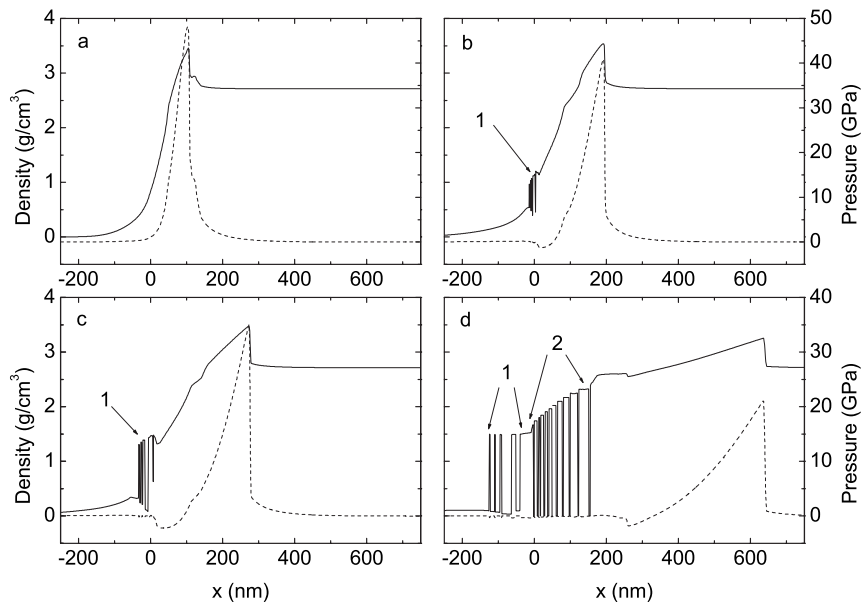

FIG. 2. Contour plots of density (solid) and pressure of heavy particles (dashed) for different time delays after irradiation: (a) $10 \mathrm{ps}$, (b) $20 \mathrm{ps,} \mathrm{(c)} 30 \mathrm{ps}$, and (d) $80 \mathrm{ps}$. Here, 1 is thermal decomposition zone and 2 is mechanical fragmentation zone. Laser parameters are the same as in Fig. 1.

ceeded, the material is decomposed into droplets and chunks (separated by vacuum). This mechanical decomposition, described also in Refs. 17, 20, and 21, concerns the major part of the ablated material in our simulations.

The dynamics of the target surface layers decomposition is presented in Fig. 2. Initially, the solid-vacuum interface is located at $x=0 \mathrm{~nm}$ and target occupies $x \geqslant 0 \mathrm{~nm}$ half space. During the collisions of heated electrons with the lattice, its temperature and pressure grow, and thus shock wave forms and propagates into the target (pressure peaks in Fig. 2). Pressure release on the free surface of the target results in material expansion in the opposite direction through a rarefaction wave. At a delay $t \sim 10 \mathrm{ps}$ after the beginning of the laser pulse, the density profile is still continuous [Fig. 2(a)]. At $t \sim 20 \mathrm{ps}$, the trajectories originated from the skin layer reach the binodal curve and penetrate into the metastable liquid region, where thermal decomposition of substance starts [Fig. 2(b)]. This process is completed by $t \sim 30$ ps [Fig. 2(c)]. Then, only mechanical effects, caused by the tensile stress (negative pressure), are involved in the process of decomposition [Fig. 2(d)]. By the time $t \sim 80 \mathrm{ps}$, the decomposition of the liquid phase is completed, because the tensile pressure wave intensity drops owing to the energy expenditure on fragmentation [compare minimal pressures in Figs. 2(c) and 2(d) at $x \sim 40 \mathrm{~nm}$ and $x \sim 260 \mathrm{~nm}$, respectively]. Completion of the mechanical decomposition corresponds to the final vibrations of the trajectories from 50 to $130 \mathrm{~nm}$ in Fig. 1 around isobar with zero pressure, similar to recent MD analysis ${ }^{21}$ and hydrodynamic simulations. ${ }^{8}$

The fractions of the target material ablated due to the described mechanisms depend both on the material and on laser parameters. At very small fluences $\left(F<0.25 \mathrm{~J} / \mathrm{cm}^{2}\right.$ for aluminum), only melting occurs. When the laser fluence is slightly above this value, material fragmentation takes place, in agreement with the previous MD simulations. ${ }^{12,20}$ If we further increase laser fluence, both mechanical and thermal mechanisms play a role. At larger $F$, all three mechanisms, (i) atomization, (ii) thermal decomposition (critical-point 


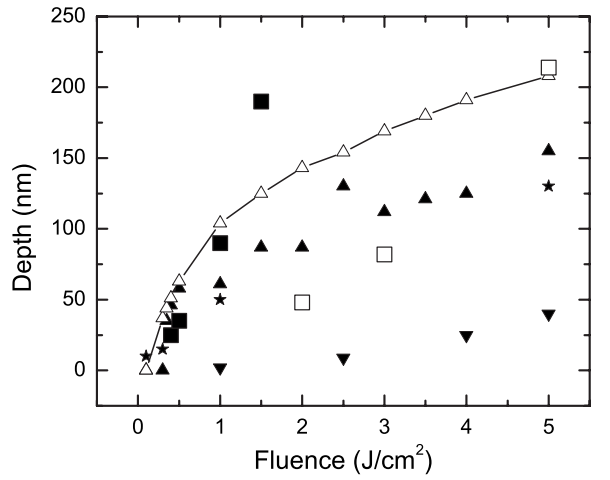

FIG. 3. Present simulation: melted $(\triangle$, solid line) and ablated $(\boldsymbol{\Delta})$ depths as a function of laser fluence for aluminum. Experimental data are taken from Ref. 5 (ם) and Ref. 7 ( $\square$ ). The errors of the experimental data were reported to be of the order of $5-10 \%$. Results of simulation taken from Ref. $11(\star)$ and Ref. $15(\boldsymbol{\nabla})$ are also shown.

phase separation), and (iii) mechanical decomposition (dynamic fragmentation), occur in different layers of the target. The third mechanism, however, is found to be dominant in our simulations, accounting about $80 \%$ of ablated mass at all considered laser fluences (from 0.1 to $5 \mathrm{~J} / \mathrm{cm}^{2}$ ).

Finally, we propose several predictions from our simulations and compare our results with the available experimental findings ${ }^{5,7}$ and simulations. ${ }^{11,15}$ We use the results of two experiments $^{5,7}$ for the model verification, though both the oxidation of the aluminum target and different techniques used in the measurements apparently result in the essential discrepancy in the experimental findings (see Fig. 3). It turns out, nevertheless, that the consideration of only critical-point phase separation effect in the calculations ${ }^{15}$ gives rise to a significant underestimation of the ablation depth (several times) with respect to the experimental values (Fig. 3). This underestimation can be explained by the fact that only 10$20 \%$ of the target material is ablated due to these critical effects, whereas most of the ablated material is ejected due to the mechanical fragmentation of the liquid phase. These results confirm that the decomposed melted zone contributes strongly into the estimation of the ablated depth.

\section{CONCLUSION}

In conclusion, we have presented the results of the hydrodynamic calculations of femtosecond laser ablation with a thermodynamically complete equation of state and with interplay between both kinetic lifetime of metastable liquid state and mechanical time to fracture. Ablation mechanisms have been investigated as a function of laser fluence. A correlation between the melted and ablated depths has been revealed. Mechanical decomposition of a metastable liquid layer has been shown to be the dominant ablation mechanism. In the present work, we have considered only aluminum ablation in one dimension. Similar analysis can be performed for different materials. In addition, an extension of the model to a two-dimensional case with multilayer materials is under way.

\section{ACKNOWLEDGMENTS}

This work was supported by the Russian Foundation for Basic Research (Project No. 06-02-17464) and the Council on Grants from the President of the Russian Federation (Project No. NSh-3683.2006.2). M.E.P. gratefully acknowledges the financial support from the CNRS, France.
${ }^{1}$ Short Pulse Laser Interactions with Matter: An Introduction, edited by P. Gibbon (Imperial College Press, London, 2005).

${ }^{2}$ Femtosecond Laser Spectroscopy, edited by Peter Hannaford (Springer, New York, 2004).

${ }^{3}$ Femtosecond Technology for Technical and Medical Applications, Edited by F. Dausinger, F. Lichtner, and H. Lubatschowski (Springer, Berlin, 2004).

${ }^{4}$ S. Nolte, B. N. Chichkov, H. Welling, Y. Shani, K. Lieberman, and H. Terkel, Opt. Lett. 24, 914 (1999).

${ }^{5}$ S. Amoruso, R. Bruzzese, M. Vitiello, N. N. Nedialkov, and P. A. Atanasov, J. Appl. Phys. 98, 044907 (2005).

${ }^{6}$ A. Plech, V. Kotaidis, M. Lorenc, and J. Boneberg, Nat. Phys. 2, 44 (2006).

${ }^{7}$ J. P. Colombier, P. Combis, F. Bonneau, R. Le Harzic, and E. Audouard, Phys. Rev. B 71, 165406 (2005).

${ }^{8}$ J. P. Colombier, P. Combis, A. Rosenfeld, I. V. Hertel, E. Audouard, and R. Stoian, Phys. Rev. B 74, 224106 (2006).

${ }^{9}$ B. Rethfeld, K. Sokolowski-Tinten, D. von der Linde, and S. I. Anisimov, Phys. Rev. B 65, 092103 (2002).

${ }^{10}$ S. I. Anisimov and B. S. Luk'yanchuk, Phys. Usp. 45, 293 (2002).

${ }^{11}$ A. M. Komashko, M. D. Feit, A. M. Rubenchik, M. D. Perry, and
P. S. Banks, Appl. Phys. A: Mater. Sci. Process. 69, S95 (1999). ${ }^{12}$ D. S. Ivanov and L. V. Zhigilei, Phys. Rev. B 68, 064114 (2003).

${ }^{13}$ S. I. Anisimov, B. L. Kapeliovich, and T. L. Perel'man, Sov. Phys. JETP 39, 375 (1974).

${ }^{14}$ K. Eidmann, J. Meyer-ter-Vehn, T. Schlegel, and S. Hüller, Phys. Rev. E 62, 1202 (2000).

${ }^{15}$ F. Vidal, T. W. Johnston, S. Laville, O. Barthélemy, M. Chaker, B. Le Drogoff, J. Margot, and M. Sabsabi, Phys. Rev. Lett. 86, 2573 (2001).

${ }^{16}$ T. E. Glover, J. Opt. Soc. Am. B 20, 125 (2003).

${ }^{17}$ L. V. Zhigilei and B. J. Garrison, J. Appl. Phys. 88, 1281 (2000).

${ }^{18}$ B. J. Garrison, T. E. Itina, and L. V. Zhigilei, Phys. Rev. E 68, 041501 (2003).

${ }^{19}$ C. Schäfer, H. M. Urbassek, and L. V. Zhigilei, Phys. Rev. B 66, 115404 (2002).

${ }^{20}$ D. Perez and L. J. Lewis, Phys. Rev. B 67, 184102 (2003).

${ }^{21}$ P. Lorazo, L. J. Lewis, and M. Meunier, Phys. Rev. B 73, 134108 (2006).

${ }^{22}$ D. E. Grady, J. Mech. Phys. Solids 36, 353 (1988).

${ }^{23}$ G. I. Kanel, S. V. Rasorenov, and V. E. Fortov, J. Appl. Mech. Tech. Phys. 25, 701 (1984).

${ }^{24}$ G. H. Miller and E. G. Puckett, J. Comput. Phys. 128, 134 
(1996).

${ }^{25}$ M. E. Povarnitsyn, T. E. Itina, P. R. Levashov, and K. V. Khishchenko, Appl. Surf. Sci. 253, 6343 (2007).

${ }^{26}$ S. I. Anisimov and B. Rethfeld, Proc. SPIE 3093, 192 (2002).

${ }^{27}$ K. V. Khishchenko, in Physics of Extreme States of Matter-2005 (IPCP RAS, Chernogolovka, 2005), pp. 170-172 (in Russian).

${ }^{28}$ L. D. Landau and E. M. Lifshits, Statistical Physics (Pergamon, Oxford, 1980).

${ }^{29}$ K. V. Khishchenko, S. I. Tkachenko, P. R. Levashov, I. V. Lomonosov, and V. S. Vorob'ev, Int. J. Thermophys. 23, 1359
(2002).

${ }^{30}$ V. I. Oreshkin, R. B. Baksht, N. A. Ratakhin, A. V. Shishlov, K. V. Khishchenko, P. R. Levashov, and I. I. Beilis, Phys. Plasmas 11, 4771 (2004).

${ }^{31}$ J. Frenkel, Kinetic Theory of Liquids (Clarendon, Oxford, 1946).

${ }^{32}$ V. P. Skripov, Metastable Liquids (Wiley, New York, 1974).

${ }^{33}$ S. I. Tkachenko, V. S. Vorob'ev, and S. P. Malyshenko, J. Phys. D 37, 495 (2004).

${ }^{34}$ V. K. Semenchenko, Surface Phenomena in Metals and Alloys (Pergamon, New York, 1961). 